\title{
Increased prediction value of biomarker combinations for the conversion of mild cognitive impairment to Alzheimer's dementia
}

Aonan Zhao ${ }^{1 \dagger}$, Yuanyuan $\mathrm{Li}^{1 \dagger}$, Yi Yan', Yinghui Qiu', Binyin $\mathrm{Li}^{1}$, Wei Xu', Ying Wang ${ }^{1}$, Jun $\mathrm{Liu}^{1,2^{*}}$ and Yulei Deng ${ }^{1,2^{*}}$ (D)

\begin{abstract}
Background: Progression of mild cognitive impairment (MCl) to Alzheimer's disease (AD) dementia can be predicted by clinical features and a combination of biomarkers may increase the predictive power. In the present study, we investigated whether the combination of olfactory function and plasma neuronal-derived exosome (NDE) $A \beta_{1-42}$ can best predict progression to $A D$ dementia.
\end{abstract}

Methods: $87 \mathrm{MCl}$ patients were enrolled and received the cognitive assessment at 2-year and 3-year follow-up to reevaluate cognition. In the meanwhile, 80 healthy controls and $88 \mathrm{AD}$ dementia patients were enrolled at baseline as well to evaluate the diagnose value in cross-section. Olfactory function was evaluated with the sniffin sticks (SS16) and $A \beta_{1-42}$ levels in NDEs were determined by ELISA. Logistic regression was performed to evaluate the risk factors for cognitive decline in $\mathrm{MCl}$ at 2-year and 3-year revisits.

Results: In the cross cohort, lower SS-16 scores and higher $A \beta_{1-42}$ levels in NDEs were found in MCI and AD dementia compared to healthy controls. For the longitudinal set, $8 \mathrm{MCl}$ individuals developed AD dementia within 2 years, and $16 \mathrm{MCl}$ individuals developed AD dementia within 3 years. The two parameter-combination of SS-16 scores and $A \beta_{1-42}$ level in NDEs showed better prediction in the conversion of $M C l$ to $A D$ dementia at 2-year and 3-year revisit. Moreover, after a 3-year follow-up, SS-16 scores also significantly predicted the conversion to AD dementia, where lower scores were associated with a 10-fold increased risk of developing AD dementia $(p=0.006)$. Similarly, higher $A \beta_{1-42}$ levels in NDEs in patients with $M C l$ increased the risk of developing AD dementia by 8.5fold $(p=0.002)$.

Conclusion: A combination of two biomarkers of NDEs $\left(A \beta_{1-42}\right)$ and SS-16 predicted the conversion of $\mathrm{MCI}$ to $\mathrm{AD}$ dementia more accurately in combination. These findings have critical implications for understanding the pathophysiology of AD dementia and for developing preventative treatments for cognitive decline.

Keywords: Alzheimer's disease, Mild cognitive impairment, Olfactory function, Neuronal-derived exosomes

\footnotetext{
* Correspondence: jly0520@hotmail.com; dyl10786@rjh.com.cn

${ }^{\dagger}$ Aonan Zhao and Yuanyuan Li contributed equally to this work.

'Department of Neurology and Institute of Neurology, Ruijin Hospital

affiliated to Shanghai Jiao Tong University School of Medicine, Shanghai,

China

Full list of author information is available at the end of the article
}

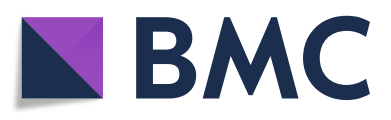

(- The Author(s). 2020 Open Access This article is licensed under a Creative Commons Attribution 4.0 International License, which permits use, sharing, adaptation, distribution and reproduction in any medium or format, as long as you give appropriate credit to the original author(s) and the source, provide a link to the Creative Commons licence, and indicate if changes were made. The images or other third party material in this article are included in the article's Creative Commons licence, unless indicated otherwise in a credit line to the material. If material is not included in the article's Creative Commons licence and your intended use is not permitted by statutory regulation or exceeds the permitted use, you will need to obtain permission directly from the copyright holder. To view a copy of this licence, visit http://creativecommons.org/licenses/by/4.0/. The Creative Commons Public Domain Dedication waiver (http://creativecommons.org/publicdomain/zero/1.0/) applies to the data made available in this article, unless otherwise stated in a credit line to the data. 


\section{Introduction}

Mild cognitive impairment (MCI), the stage between normal aging and Alzheimer's disease (AD) dementia, is associated with a higher risk of dementia [1, 2]. A recent meta-analysis indicated that about $45 \%$ of MCI patients maintained stable, whereas $28 \%$ progressed to $\mathrm{AD}$ and $15 \%$ return to normal status without recurrence [3]. Recent work has aimed to improve the detection of the early stages in AD dementia and improve the methods used to identify individuals with MCI who are at high risk of developing $\mathrm{AD}$ dementia.

Olfactory dysfunction has been identified in patients with AD dementia [4]. In a longitudinal study, olfactory impairment was used as a biomarker for diagnosing MCI and AD dementia, predicting the progression of $\mathrm{AD}$ dementia in normally-aging individuals [5]. However, odor identification tests are not specific to AD dementia and may also be impaired in Parkinson's disease and other forms of dementia [6]. Given that olfactory function appears to be altered across neurodegenerative diseases and is also affected by smoking habits and respiratory diseases [7], more sensitive and specific biomarkers are needed to assist odor identification tests as a clinical diagnostic role of AD dementia.

Amyloid beta $(A \beta)$, AD-specific pathological changes, are deposited in the olfactory bulb or other brain regions related to olfactory function [8]. $A \beta_{1-40}$ and $A \beta_{1-42}$ in cerebrospinal fluid (CSF) have consistently been shown to predict the conversion from MCI to AD dementia [9]. However, the measurement of CSF biomarkers is invasive and discrepant findings have been reported when attempting to use a combination of plasma proteins to predict AD dementia progression in stable MCI patients [10]. Hence, a more accurate measurement for $A \beta_{1-42}$ with better clinical practicality is in need. Neuronalderived exosomes (NDEs) in plasma are released from neurons, reported to contain amyloid-beta precursor protein and $A \beta$, released from the central nervous system (CNS) [11]. A $\beta$, contained within NDEs, isolated from plasma, accurately predicted the development of $\mathrm{AD}$ dementia up to 5 years before AD onset [12] . Therefore, the need to recognize $\mathrm{AD}$ dementia at an early or more treatable stage promotes the study of exosome biomarkers. The evaluation of olfactory function combined with $A \beta$ in NDEs may lead to new approaches in predicting the risk of MCI to AD dementia.

However, no studies to date have investigated whether the combination of $A \beta$ and olfactory test can improve predicting the transition risk of MCI to AD dementia. Firstly, we aimed to measure $A \beta$ levels from plasma NDEs and odor identification in AD dementia and MCI groups. Secondly, this longitudinal study focused on patients with MCI who progressed to a probable AD dementia within 3 years after baseline (called MCI converters (MCI-c)) and compared them with clinically stable patients who did not develop to AD dementia (called MCI non-converters (MCI-nc)). The olfactory test and the neurogenic exosomes $\left(A \beta_{1-42} \mathcal{E} A \beta_{1-40}\right)$ were performed to investigate baseline differences between MCI converters and non-converters. The purpose of the research was to establish a predictive model identifying individuals with MCI who are at risk of developing $\mathrm{AD}$ dementia. We hypothesized that a combination of the sniffin sticks (SS-16) and plasma NDEs Aßhelped screen and MCI patients at higher risk of cognitive decline, which can benefit from early intervention to prevent the risk of disease progression.

\section{Subjects and methods}

\section{Study population and clinical profiling}

Participants were recruited from the neurology clinic at Ruijin Hospital from October 2015 to May 2019. All volunteers gave their informed, written consent prior to study participation. This study was approved by the Research Ethics Committee of Ruijin Hospital. All patients with $\mathrm{AD}$ dementia were diagnosed as probable AD dementia following the National Institute on Aging and Alzheimer's Association (NIA-AA) diagnostic guidelines for probable $A D$ dementia with support of structural MRI images [13]. To ensure volunteers understood the task, only patients with mild to moderate AD dementia $(24 \geq$ Mini Mental State Examination (MMSE) $\geq 10)$ participated on the odor identification tests. MCI with deficits in memory function were diagnosed according to the Mayo Clinic criteria [14, 15]. The criteria include subjective memory complaint corroborated by an informant together with preserved everyday activities, a memory impairment based on a standard neuropsychological test, preserved global cognitive functions and finally the exclusion of dementia. The healthy control subjects were age-, sex-, and education-matched and were recruited from the local community in Shanghai. Inclusion criteria for normal controls required a MMSE score $\geq 28$ without any memory-related complaint. Subjects with the presence of dementia or other neurological diseases such as Parkinson's disease were excluded. Besides, participants were excluded if they have any of the following medical problems: acute diabetic complications, history of acute cerebrovascular accident, history of acute cardiovascular accident, systemic disorders such as malignancy and lupus which were not cured, severe infection, drug abuse or dependency condition and severe psychiatric disorders which were not cured. In this study, we excluded participants with possible factors impairing olfactory function, such as chronic rhinitis, recent upper respiratory infections, and nose surgery.

All participants completed the neuropsychological battery including the MMSE [16], the Montreal Cognitive 
Assessment (MoCA) [14], Auditory Verbal Learning Test (AVLT) [17], Alzheimer's Disease Assessment Scale cognitive subscale (ADAS-cog),Zung Self-rating the Anxiety Scale (SAS) and the Zung Self-rating Depression Scale (SDS) [18]. All tests were administered by memory-related specialists with professional training. Experienced neurologists performed all diagnoses based on a thorough review of the patient's medical history, neurological examinations, laboratory tests and structural MRI results. All participants in the study $(n=255)$ including 80 healthy controls, 87 individuals with MCI and 88 patients with AD dementia completed the SS-16 test and the tests measuring neuronal-derived exosomes at baseline. Follow-up visiting started from January 2017 to May 2019, where the mean follow-up time was 34.7 months. During the 2-year and 3-year follow-ups, patients with MCI were reclassified as MCI converters (MCI-c) or MCI non-converters (MCI-nc) based on whether they had been diagnosed with AD dementia.

\section{SS-16 assessment}

In the study, the SS-16 was selected as an evaluation instrument to verify the sensitivity and feasibility of the odor identification test. The Chinese version of the SS16 was validated with Chinese patients with Parkinson's disease in 2012 [19]. Trained specialists, who were blinded to the diagnosis of the volunteer, administered the 16-item odor identification tests. Participants were required to place the odor sticks $2 \mathrm{~cm}$ away from their nose and were instructed to smell the stick for $3 \mathrm{~s}$. Following this, volunteers were instructed to identify the odor using a multiple-choice question with 4 possible answers. The time interval between the presentation of each odor was approximately $30 \mathrm{~s}$. Each correct answer was assigned one point and the total score varied from 0 to the highest possible of 16 .

\section{Isolation of neuronal-derived exosomes (NDEs) from plasma}

L1 cell adhesion molecule (L1CAM) is a member of cell adhesion molecules primarily expressed in the nervous system and is proved to be a marker on the surface of exosomes that are specifically derived from the neurons $[20,21]$. Exosomes were collected from plasma and the content of those originating from neurons (NDEs) were enriched by absorption with the anti-L1CAM antibody. Overall, $500 \mu \mathrm{L}$ plasma was incubated with thromboplastin-D (Fisher Scientific, Inc., Hanover Park, IL) followed by calcium- and magnesium-free Dulbecco balanced salt solution with protease inhibitor cocktail (Roche Applied Sciences, Inc., Indianapolis, IN) and phosphatase inhibitor cocktail (Pierce Halt, Thermo Scientific, Inc., Rockford, IL). After centrifugation, supernatants were incubated with ExoQuick exosome precipitation solution (EXOQ; System Biosciences, Inc., Mountain View, CA) and resultant suspensions centrifuged at $1500 \times \mathrm{g}$ for $30 \mathrm{~min}$ at $4{ }^{\circ} \mathrm{C}$ [22]. Each pellet was re-suspended in $200 \mu \mathrm{L}$ of distilled water with inhibitor cocktails followed by immunochemical enrichment of exosomes from neural sources.

Total exosome suspensions were incubated with $2 \mu \mathrm{g}$ of mouse anti-human L1CAM (neural adhesion protein) biotinylated antibody (Abcam, Cambridge, MA, USA) in $50 \mu \mathrm{L}$ of $3 \% \mathrm{BSA}$ for $60 \mathrm{~min}$ at $20^{\circ} \mathrm{C}$ followed by addition of $10 \mu \mathrm{L}$ of Streptavidin-Plus UltraLink resin (Pierce-Thermo Scientific, Inc.) in $40 \mu \mathrm{L}$ of $3 \%$ BSA and further incubation for $60 \mathrm{~min}$ [19]. After centrifugation at $400 \times \mathrm{g}$ for $5 \mathrm{~min}$ at $4{ }^{\circ} \mathrm{C}$, pellets were re-suspended in $50 \mu \mathrm{L}$ of $0.05-\mathrm{M}$ glycine- $\mathrm{HCl}$ (pH 3.0), incubated at $4{ }^{\circ} \mathrm{C}$ for $10 \mathrm{~min}$, and re-centrifuged. Each supernatant was transferred to a new Eppendorf tube containing $5 \mu \mathrm{L}$ of $1-\mathrm{M}$ Tris- $\mathrm{HCl}$ (pH 8.0) mixed with $0.50 \mathrm{~mL}$ M-PER mammalian protein extraction reagent (Thermo Scientific, Inc.), containing protease and phosphatase inhibitors, and mixed and stored at $-80^{\circ} \mathrm{C}$.

\section{Quantification of NDE and ELISA assay}

L1CAM-positive NDE cargo proteins were quantified by using the human-specific ELISAs for $A \beta_{1-42}$ (Anogen, Ontario, CA), $\mathrm{A} \beta_{1-40}$ (Anogen, Ontario, CA) and ExoELISA CD63 Kit (System Biosciences, Inc., Mountain View, CA) in duplicate with verification of bicinchoninic acid (BCA) reagent-based protein quantitation (Thermo Scientific, Inc.) to normalize the relative values for each sample.

L1CAM-positive plasma NDEs were characterized based on size and shape using transmission electron microscopy (TEM). The degree of purity was verified by western blot with positive exosomal marker CD63 (Abcam, Cambridge, MA, USA) and Tsg101 (Abcam, Cambridge, MA, USA) and negative exosomal marker GM130 (Abcam, Cambridge, MA, USA). The size of the samples was directly determined by NTA using a NanoSight LM10 microscope (NanoSight Ltd., Salisbury, UK).

\section{ApoE $\varepsilon 4$ genotype}

Genomic DNA was extracted from peripheral blood through the standardized phenol/chloroform extraction method. ApoE $\varepsilon 2 / 3 / 4$ alleles were determined by the following primers to detect rs7412 and rs429358. Forward primer: AGGAACAACTGACCCCGGTG; Reverse Primer: GCTGCCCATCTCCTCCATCC. All subjects were classified as ApoE $\varepsilon 4$ carriers with APOE $\varepsilon 2 / \varepsilon 4, \varepsilon 3 / \varepsilon 4$ and $\varepsilon 4 / \varepsilon 4$ or as ApoE $\varepsilon 4$ non-carriers with APOE $\varepsilon 2 / \varepsilon 2$, $\varepsilon 2 / \varepsilon 3$ and $\varepsilon 3 / \varepsilon 3$.

\section{Statistical analysis}

Statistical analyses were conducted with SPSS (version 19.1; IBM Corp., Armonk, NY). The significance level 
was set at $p<0.05$. One-way ANOVAs with the least significant difference (LSD) and post-hoc tests were used to compare differences between the three groups (AD dementia, MCI, and healthy controls). We used chisquared and split chi-squared tests to identify differences in sex, education levels, smoking status and the accuracy of detecting the 16 odors between the three groups. The Pearson correlation was used to determine the association between A $\beta 1-42$, SS-16 and MMSE/MoCA scores. Receiver operating characteristic (ROC) curves were plotted for SS-16 and $A \beta_{1-42}$ in NDEs by calculating the sensitivity and specificity of their diagnostic power in $\mathrm{HC}, \mathrm{MCI}, \mathrm{AD}$ dementia, MCI-c and MCI-nc [23]. Logistic regression was used to evaluate whether biological variables (SS-16, $A \beta_{1-42}$ and ApoE $\varepsilon 4$ status) predicted the conversion to $\mathrm{AD}$ dementia in individuals with $\mathrm{MCI}$ at 2-year and 3-year follow-up. To assess how SS-16 and $\mathrm{A} \beta_{1-42}$ increase the risk of $\mathrm{AD}$ dementia conversion, we built a logistic regression model of the ten markers controlling for age, sex and education.

\section{Results}

Demographic and neuropsychological characteristics

Demographic features and clinical data of healthy controls and patients with $\mathrm{AD}$ dementia and $\mathrm{MCI}$ are shown in Table 1. There were no significant differences between the three groups based on age, sex, or education levels. Furthermore, we classified the enrolled subjects to be ApoE\&4 carriers or ApoE \&4 non-carriers. $39 \%$ of $\mathrm{AD}$ dementia patients, $17 \%$ of $\mathrm{MCI}$ patients and $10 \%$ of healthy individuals were positive for ApoE $\varepsilon 4$. A greater number of patients with $\mathrm{AD}$ dementia were ApoE\&4 carriers relative to controls $(p<0.001$, Table 1$)$. $59 \%$ of $\mathrm{AD}$ dementia patients were maintained on cholinesterase inhibitors with a mean dose of $5.2 \mathrm{mg}$ per day. Compared with MCI and controls, patients with AD dementia had lower scores of MMSE, MoCA, AVLT-SR and AVLT-LR $(p<0.001$, Table 1$)$, but exhibited a higher ADAS-cog score $(p<0.001$, Table 1$)$. However, MCI subjects also had lower MMSE, MoCA and AVLT scores relative to controls and greater ADAS-cog scores compared to controls $(p<0.05$; Table 1$)$. Applied a cutoff score of 1 SD under population mean standardized for age and gender according to AVLT tests [24], 74 out of the enrolled MCI patients were amnestic MCI (aMCI). Olfactory function was further assessed, and scores of SS-16 were significantly lower in AD dementia than in the control and MCI groups $(11.2 \pm 1.9$ for controls; $9.1 \pm 2.7$ for MCI; $5.9 \pm 2.7$ for AD dementia; $p<$ 0.001 ; Table 1). Moreover, lower SS-16 scores were observed in MCI subjects than in healthy controls $(p<$ $0.001)$.

Table 1 Demographic features of the participants in baseline

\begin{tabular}{|c|c|c|c|c|}
\hline Demographics & $\mathrm{HC}(\boldsymbol{n}=80)$ & $\mathrm{MCl}(\boldsymbol{n}=87)$ & AD dementia $(\boldsymbol{n}=88)$ & $p$ value \\
\hline Age(y) & $67.3(4.7)$ & $66.2(4.3)$ & $67.7(4.2)$ & 0.785 \\
\hline \multicolumn{5}{|l|}{ Sex } \\
\hline Female & $44(55 \%)$ & $47(54 \%)$ & $50(47 \%)$ & \multirow[t]{2}{*}{0.931} \\
\hline Male & $36(45 \%)$ & $40(46 \%)$ & 38 (53\%) & \\
\hline Education duration(y) & $10.8(2.9)$ & $10.5(2.6)$ & $10.4(2.5)$ & 0.196 \\
\hline \multicolumn{5}{|l|}{ ApoE $\varepsilon 4$ carrier } \\
\hline$(+)$ & $8(10 \%)$ & $15(17 \%)$ & $34(39 \%)$ & \multirow[t]{2}{*}{$0.000^{b, c}$} \\
\hline$(-)$ & $73(90 \%)$ & $72(83 \%)$ & $54(61 \%)$ & \\
\hline Mean ChEl dose (mg) & / & / & $5.2(2.3)$ & / \\
\hline MMSE & $29.3(0.7)$ & $25.7(1.4)$ & $17.0(2.1)$ & $0.000^{a, b, c}$ \\
\hline MoCA & $26.4(1.3)$ & $21.6(1.8)$ & $11.2(2.5)$ & $0.000^{a, b, c}$ \\
\hline SAS & $27.4(3.7)$ & $27.6(4.5)$ & $27.5(4.6)$ & 0.986 \\
\hline SDS & $29.4(6.4)$ & $29.1(6.7)$ & $29.6(6.5)$ & 0.915 \\
\hline ADAS-cog & $7.4(3.8)$ & $11.8(4.4)$ & $21.4(5.2)$ & $0.000^{a, b, c}$ \\
\hline AVLT-SR & $7.6(1.3)$ & $5.2(2.1)$ & $2.3(1.2)$ & $0.000^{a, b, c}$ \\
\hline AVLT-LR & $7.3(1.4)$ & $4.8(1.6)$ & $1.7(1.1)$ & $0.000^{a, b, c}$ \\
\hline SS-16 & $11.2(1.9)$ & $9.1(2.7)$ & $5.9(2.7)$ & $0.000^{a, b, c}$ \\
\hline
\end{tabular}

"a" means $\mathrm{HC}$ group and $\mathrm{MCl}$ group are significantly different

"b" means HC group and AD dementia group are significantly different

" $\mathrm{C}$ " means $\mathrm{MCl}$ group and $\mathrm{AD}$ dementia group are significantly different

Abbreviations: MMSE Mini Mental State Examination, MoCA Montreal Cognitive Assessment, SAS Zung Self-rating the Anxiety Scale, SDS Zung Self-rating Depression Scale, ADAS-cog Alzheimer's Disease Assessment Scale-cognitive subscale, AVLT Auditory Verbal Learning test, SS-16 the 16-item odor identification test from Sniffin Sticks, $H C$ healthy control, $M C l$ mild cognitive impairment, AD Alzheimer's disease, ChEl Cholinesterase inhibitor 


\section{$A \beta_{1-42}$ in plasma NDEs was elevated in $\mathrm{MCl}$ and $\mathrm{AD}$ dementia patients}

Plasma NDEs were first analyzed for morphology and size distribution using TEM (Fig. 1a), which revealed a population of morphologically distinctive particles of approximately 100-nm diameter, as previously reported [25]. The purity of plasma NDEs was also validated with western blot by three positive exosomal markers (L1CAM, CD63, and Tsg101) and one negative exosomal marker (GM130) (Fig. 1b). In Fig. 1c, the size of the exosomes was directly determined by NTA. Moreover, by performing the ELISA assay, we found the expression of $A \beta_{1-42}$ among three groups based on the consistent distribution of CD63 (Fig. 1d). As shown in Fig. 1e, AD dementia patients exhibited significantly higher concentrations of $A \beta_{1-42}$ in NDE compared to healthy and MCI volunteers $(26.0 \pm 16.8 \mathrm{pg} / \mathrm{ml}$ for $\mathrm{AD}$ dementia; $14.0 \pm 9.3 \mathrm{pg} / \mathrm{ml}$ for $\mathrm{MCI}, 8.4 \pm 3.9 \mathrm{pg} /$ $\mathrm{ml}$ for controls, $p<0.001)$. Moreover, MCI patients had greater concentrations of $A \beta_{1-42}$ relative to healthy volunteers $(p=0.001)$. However, there was no significant difference in the $A \beta_{1-40}$ levels among the three groups in Fig. 1f. As for the ratio of $A \beta_{1-42} / A \beta_{1-40}, A D$ dementia patients showed elevated $A \beta_{1-42} / A \beta_{1-40}$ ratios than MCI and $\mathrm{HC}$ groups $(0.13 \pm 0.08$ for $\mathrm{AD}$ dementia; $0.07 \pm 0.04$ for MCI, $0.05 \pm 0.03$ for controls, $p<0.001$ ) in Fig. $1 \mathrm{~g}$.
There was only an increasing trend but less statistical significance compared the $\mathrm{MCI}$ and $\mathrm{HC}$ groups $(p=0.057)$.

\section{Evaluation of the $\mathrm{MCl}$ converters and non-converters} In total, $78 \mathrm{MCI}$ patients completed both the 2 years and 3 years follow-up questionnaires. The mean followup time for 2 years and 3 years revisits were $24.3 \pm 2.1$ and $34.7 \pm 3.2$ months. 8 of $78 \mathrm{MCI}$ patients (10.3\%) developed $\mathrm{AD}$ dementia after a median of 2 years of prospective follow-up; whereas 16 of $78 \mathrm{MCI}$ (20.5\%) developed AD dementia after a median of 3 years of prospective follow-up. Demographic characteristics of the 3 years' follow-up visit between MCI converters and nonconverters are described in Table 2. All the MCI-c patients met the criteria for probable AD dementia. The MCI-c patients had significantly lower scores on the MMSE, MoCA and AVLT tests than the MCI-nc patients $(p<0.001)$. There were no significant differences in age, sex, or education between the non-converters and converters.

\section{Risk of SS-16 and $A \beta 1-42$ NDEs in predicting $\mathrm{MCl}$ conversion}

In terms of the predictive power of $A \beta_{1-42}$ and SS- 16 for $\mathrm{AD}$ dementia, ROC analysis was further conducted to

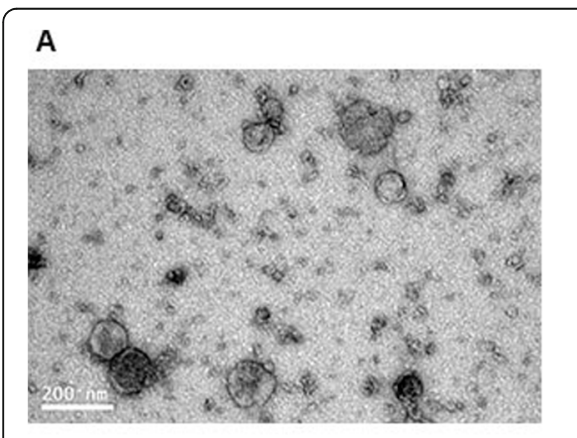

B

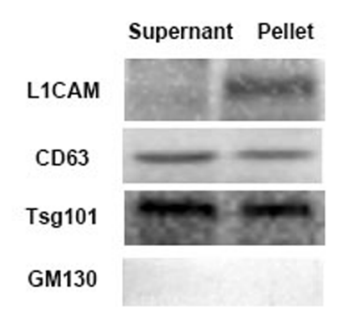

C

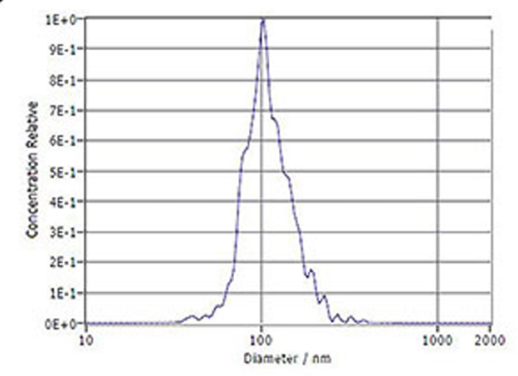

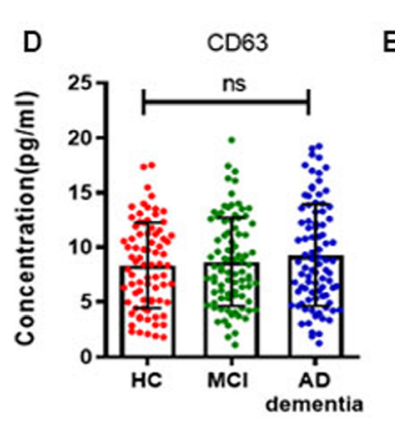

E

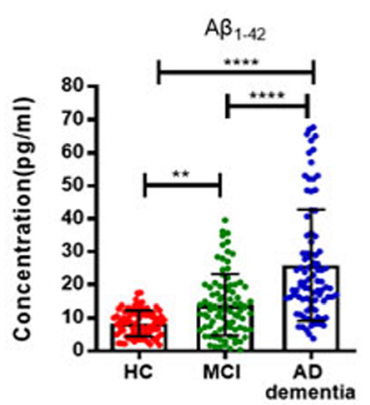

$\mathbf{F}$
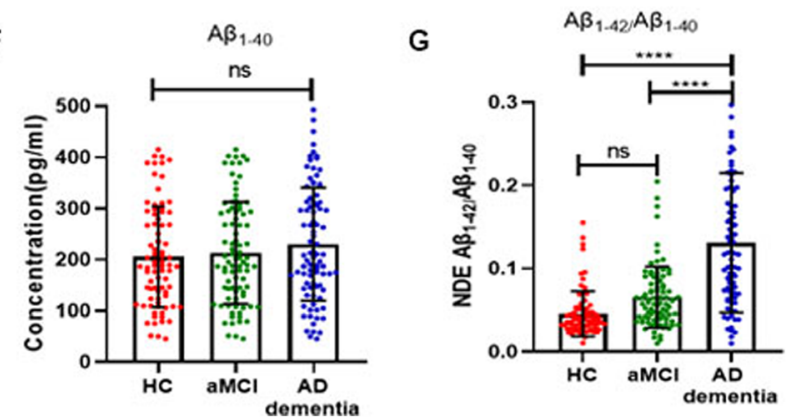

Fig. 1 Identification and quantification of neuronal-derived exosomes (NDEs). a. Electron microscopic image showing that NDEs were successfully collected. b. Western blot showing that exosomal positive marker L1CAM, CD63, Tsg101 was highly expressed in exosomal pellet but not detected in supernatants. c.NTA analysis of concentration and diameter in NDEs. d.ELISA test of CD63 level in NDEs indicating that the number of exosomes was not significantly different in three groups. e. ELISA test of $A \beta_{1-42}$ level in NDEs showing that $A \beta_{1-42}$ was elevated in $M C l$ and $A D$ dementia groups. **: $p<0.01$; ${ }^{* * *}: p<0.001$. f. ELISA test of $A \beta_{1-40}$ level in NDEs indicating that $A \beta_{1-40}$ was not significantly different in three groups. g. ELISA test of $A \beta_{1-42} / A \beta_{1-40}$ level in NDEs indicating that $A \beta_{1-42} / A \beta_{1-40}$ level was elevated in $M C l$ and $A D$ dementia groups. $* * * *: p<0.001$ 
Table 2 Characteristics of $\mathrm{MCl}$ non-convertors and convertors in three years follow-up

\begin{tabular}{llll}
\hline Demographics & MCl-nc $(\boldsymbol{n}=62)$ & $\mathrm{MCl}$-c $(\boldsymbol{n}=16)$ & $\boldsymbol{p}$ value \\
\hline Age, y & $68.3(4.1)$ & $68.5(3.8)$ & 0.844 \\
Sex & & & \\
$\quad$ Female & $34(55 \%)$ & $9(56 \%)$ & 0.919 \\
$\quad$ Male & $28(45 \%)$ & $7(44 \%)$ & \\
Education duration, y & $10.4(2.7)$ & $10.3(1.9)$ & 0.953 \\
ApoE \&4 carrier & & & \\
$\quad(+)$ & $10(16 \%)$ & $4(25 \%)$ & 0.410 \\
$\quad(-)$ & $52(84 \%)$ & $12(75 \%)$ & \\
MMSE & $25.8(1.4)$ & $19.3(1.7)$ & $\mathbf{0 . 0 0 0}$ \\
MoCA & $22.3(1.7)$ & $14.5(1.5)$ & $\mathbf{0 . 0 0 0}$ \\
ADAS-cog & $19.2(3.6)$ & $30.0(3.3)$ & $\mathbf{0 . 0 0 0}$ \\
AVLT-SR & $5.7(1.4)$ & $2.8(1.3)$ & $\mathbf{0 . 0 0 0}$ \\
AVLT-LR & $4.1(1.3)$ & $1.5(1.0)$ & $\mathbf{0 . 0 0 0}$ \\
\hline Abbrvitions: MMSE Min & Ment State Exminaton, MoCA Montea Cognive
\end{tabular}

Abbreviations: MMSE Mini Mental State Examination, MoCA Montreal Cognitive Assessment, ADAS-cog Alzheimer's Disease Assessment Scale-cognitive subscale, AVLT Auditory Verbal Learning test, $M C I-n c$ not converted from $\mathrm{MCl}$ to $\mathrm{AD}$ dementia, $\mathrm{MCl}-\mathrm{c}$ converted from $\mathrm{MCl}$ to $\mathrm{AD}$ dementia

determine whether they have value in predicting the conversion to $\mathrm{AD}$ dementia in $\mathrm{MCI}$ individuals. As was shown in Fig. 2 and Table 3, SS-16 and plasma $A \beta_{1-42}$ in NDEs classification demonstrated the good value of risk prediction. For the 2-year follow up, the ROC curve showed an AUC of 0.81 with a cutoff value of $8(p=$ 0.004, 95\% CI:0.66-0.96) for SS-16 and 0.84 $(p=0.002$, 95\% CI:0.72-0.95) for $A \beta_{1-42}$ in NDEs with a cutoff value of 14.02, with the combined AUC increased to 0.93 (Table 3). For the 3-year follow-up, AUC was 0.83 $(p<0.001,95 \%$ CI:0.72-0.93) for SS-16 and 0.84 ( $p=$ 0.002, 95\% CI:0.72-0.95) for $A \beta_{1-42}$ in NDEs, with the combined AUC of $0.95(p<0.001$, Table 3$)$.

To assess how SS-16 and $A \beta_{1-42}$ increase the risk of AD dementia converting, we built a logistic regression model of the ten markers controlling for age, sex and education. In the 2-year follow-up visit, MCI patients with SS-16 scores lower than 8 showed an 8.3-fold increased risk of converting to $\mathrm{AD}$ dementia $(p=0.012)$, whereas patients with higher $A \beta_{1-42}$ levels in NDE showed an 11.1-fold increased risk for developing $\mathrm{AD}$ dementia $(p=0.028$, Table 4). In the 3-year follow up, a similar trend was observed for SS-16 and $A \beta_{1-42}$, where there was a 10 -fold risk for individuals with SS-16 scores less than $8(p=0.006)$ and 8.5 -fold risk for $\mathrm{A} \beta_{1-42}(p=$ 0.002 , Table 4). There were no significant differences between ApoE $\varepsilon 4$ carriers in the 2-year or 3-year follow-up visit.

\section{Stratified analysis of SS-16 and A $\beta 1-42$ NDEs in ApoE $\varepsilon 4$ status}

According to the baseline characteristics, 14 (17.9\%) patients were ApoE $\varepsilon 4$ positive, we, therefore, investigated the potential predictors of disease progression in specific subgroups. We found that ApoE $\varepsilon 4$ non-carriers with lower SS-16 scores (OR=7.1, 95\% CI: 1.4-33.3, $p=$ 0.015; Table 5) were more likely to develop AD dementia. Moreover, patients without the ApoE $\varepsilon 4$ mutation exhibited higher $A \beta_{1-42}$ levels and showed a higher risk of developing $\mathrm{AD}$ dementia $(\mathrm{OR}=9.4,95 \% \mathrm{CI}$ : $1.9-47.8$, $p=0.007$; Table 5). However, this was not statistically significant and a larger sample size may be needed.

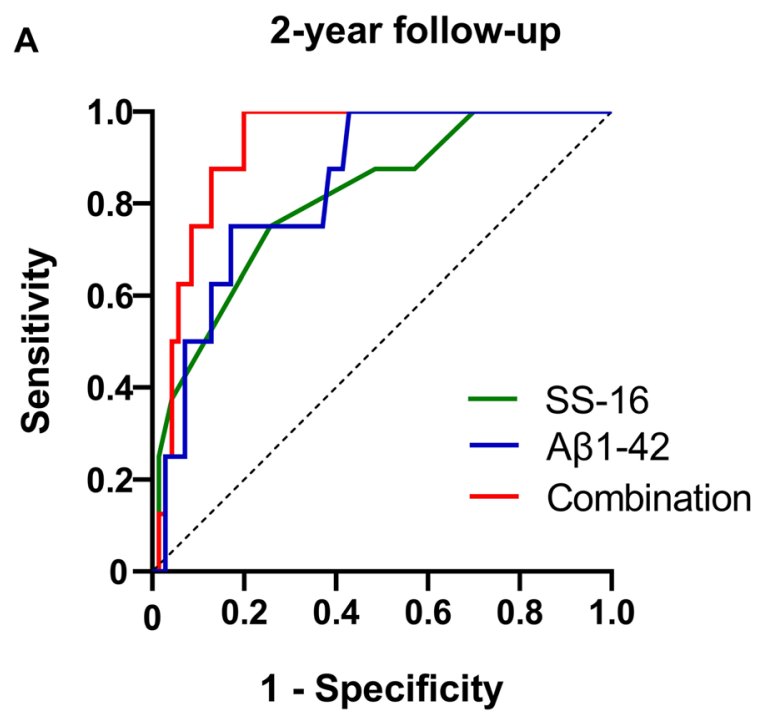

B

3-year follow-up

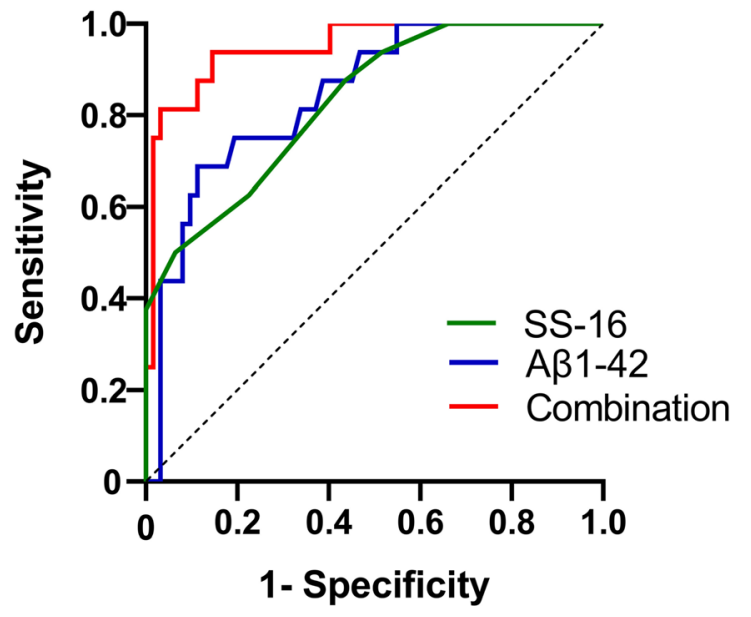

Fig. 2 High diagnostic performance of $A \beta_{1-42}$ and SS-16 for $\mathrm{MCl}$ converting to $A D$ dementia. a showed ROC analysis of $A \beta_{1-42}$ and SS-16 respectively and in combination for $\mathrm{MCl}_{-\mathrm{C}}$ and $\mathrm{MCl}-\mathrm{nc}$ in a 2-year follow-up. b showed ROC analysis of $\mathrm{A} \beta_{1-42}$ and $\mathrm{SS}-16$ respectively and in combination for $\mathrm{MCl}-\mathrm{c}$ and $\mathrm{MCl}-\mathrm{nc}$ in a 3-year follow-up 
Table 3 Characteristics of ROC curves in converted $\mathrm{MCl}$ after follow-up visit

\begin{tabular}{|c|c|c|c|c|c|c|c|c|c|c|}
\hline & \multicolumn{5}{|c|}{ two-years follow up } & \multicolumn{5}{|c|}{ three-years follow up } \\
\hline & Sensitivity & Specificity & AUC & $95 \% \mathrm{Cl}$ & $\mathrm{p}$ & Sensitivity & Specificity & AUC & $95 \% \mathrm{Cl}$ & $p$ \\
\hline SS-16 & $75.00 \%$ & $74.29 \%$ & 0.81 & $0.66-0.96$ & 0.004 & $62.50 \%$ & $77.42 \%$ & 0.83 & $0.72-0.93$ & 0.000 \\
\hline$A \beta_{1-42}$ & $87.50 \%$ & $61.43 \%$ & 0.84 & $0.72-0.95$ & 0.002 & $87.50 \%$ & $61.43 \%$ & 0.84 & $0.72-0.95$ & 0.000 \\
\hline Combination & $87.50 \%$ & $87.14 \%$ & 0.93 & $0.86-0.99$ & 0.000 & $93.75 \%$ & $85.48 \%$ & 0.95 & $0.89-1.00$ & 0.000 \\
\hline
\end{tabular}

Abbreviations: Cl confidence interval, SS-16 the 16-item odor identification test from Sniffin Sticks, ROC receiver operating characteristic, AUC area under the curve

Association between $A \beta_{1-42}$, SS-16 and cognitive function To determine the association between $A \beta_{1-42}$, SS-16 and MMSE/MoCA, correlation analysis was used. The unadjusted analyses showed a strong association between higher MMSE and MoCA score and higher SS-16 score $(p=0.002, \mathrm{r}=0.392$ for MMSE; $p=0.001, \mathrm{r}=0.453$ for MoCA; Fig. 3a) in MCI and AD dementia groups. Similarly, significant negative correlations were found between MMSE/MoCA scores and A $\beta_{1-42}$ levels $(p=0.021$, $\mathrm{r}=-0.345$ for MMSE; $p=0.007, \mathrm{r}=-0.349$ for MoCA; Fig. 3b). Interestingly, further analyses also showed an association between higher SS-16 score and reduced values of $\mathrm{A} \beta 1-42$ in NDEs $(p=0.011, \mathrm{r}=-0.442$; Fig. 3c).

Subsequently, ROC curve analysis was performed to evaluate the discriminative power of $A \beta_{1-42}$ and SS-16 in the diagnosis for $\mathrm{MCI}$ and $\mathrm{AD}$ dementia, respectively and in combination. Both $\mathrm{A} \beta_{1-42}$ (AUC: 0.69; $p<0.001$; 95\% CI: $0.61-0.77$ for MCI; AUC: $0.90 ; p<0.001$; $95 \%$ CI: $0.85-0.94$ for AD dementia, Fig. $3 \mathrm{~d}$ and e) and SS-16 have diagnostic value for individuals with MCI and patients with AD dementia (AUC: 0.65: $p=0.001$; 95\% CI: 0.56-0.73 for MCI; AUC: $0.90 ; p<0.001$; 95\% CI: $0.85-$ 0.94 for AD dementia, Fig. $3 \mathrm{~d}$ and e). Moreover, their combination resulted in a significant increase in the cstatistics of AUC: 0.71 (95\% CI: $0.63-0.79, p<0.001$ ) and AUC: 0.96 (95\% CI: 0.94-0.99, $p<0.001$ ), which showed better diagnostic efficiency for MCI or AD dementia relative to the use of each of these variables in isolation (Fig. 3d and e). Moreover, the combination of $\mathrm{A} \beta_{1-42}$ and SS-16 also showed modest accuracy in distinguishing MCI and AD dementia groups (AUC: 0.81; $p<0.001$; 95\% CI: 0.74-0.87, Fig. 3f).

\section{Discussion}

Previous studies have identified biomarkers to facilitate identifying individuals who are at risk of developing $\mathrm{AD}$ dementia. Our study was a longitudinal study that investigated whether NDEs in plasma and olfactory tests may also predict the conversion from $\mathrm{MCI}$ to $\mathrm{AD}$ dementia in a Chinese population. During the 3-year follow-up, 16 subjects developed probable AD dementia (MCI-c) and 62 did not convert to AD dementia (MCI-nc). At baseline, there were significant differences in SS-16 scores and neurogenic exosomal $\mathrm{A} \beta_{1-42}$ levels between individuals who later developed dementia relative to those who did not. Our study indicated that age, ApoE $\varepsilon 4$ status, higher-levels of $A \beta_{1-42}$ in plasma NDEs and lower SS-16 scores predicted the AD dementia conversion in individuals with MCI patients with modest accuracy.

Olfactory impairment was first reported as a clinical symptom of AD dementia more than 30 years ago [26]. Our finding that olfactory function was impaired and predicted transition is consistent with previous literature showing that olfactory deficiencies exist before patients are diagnosed with $\mathrm{AD}$ [27] and literature showing that

Table 4 Evaluation between the convertors and non-convertors

\begin{tabular}{|c|c|c|c|c|c|c|c|c|}
\hline & \multicolumn{4}{|c|}{ two-years follow up } & \multicolumn{4}{|c|}{ three-years follow up } \\
\hline & $\begin{array}{l}\mathrm{MCl}-\mathrm{nc} \\
(\boldsymbol{n}=70,90 \%)\end{array}$ & $\begin{array}{l}\mathrm{MCl}-\mathrm{C} \\
(\boldsymbol{n}=8,10 \%)\end{array}$ & $\begin{array}{l}\text { OR } \\
(95 \% \mathrm{Cl})\end{array}$ & $P$ & $\begin{array}{l}\mathrm{MCl}-\mathrm{nc} \\
(\boldsymbol{n}=62,79 \%)\end{array}$ & $\begin{array}{l}\mathrm{MCl}-\mathrm{C} \\
(\boldsymbol{n}=16,21 \%)\end{array}$ & $\begin{array}{l}\text { OR } \\
(95 \% \mathrm{Cl})\end{array}$ & $P$ \\
\hline \multicolumn{9}{|l|}{ SS-16 } \\
\hline$<8$ & $18(26 \%)$ & $6(75 \%)$ & $0.12(0.02-0.6)$ & 0.012 & $27(44 \%)$ & $14(88 \%)$ & $0.1(0.02-0.5)$ & 0.006 \\
\hline$\geq 8$ & $52(74 \%)$ & $2(25 \%)$ & & & $35(56 \%)$ & $2(12 \%)$ & & \\
\hline \multicolumn{9}{|c|}{$A \beta_{1-42}, \mathrm{pg} / \mathrm{ml}$} \\
\hline$<14.02$ & $43(61 \%)$ & $7(88 \%)$ & 11.1 (1.3-95.7) & 0.028 & $41(66 \%)$ & $3(19 \%)$ & $8.5(2.2-33)$ & 0.002 \\
\hline$\geq 14.02$ & 27 (39\%) & $1(12 \%)$ & & & $21(34 \%)$ & 13 (81\%) & & \\
\hline \multicolumn{9}{|c|}{ ApoE ع4 carrier } \\
\hline$(+)$ & $12(17 \%)$ & $2(25 \%)$ & $1.6(0.29-9.0)$ & 0.590 & $10(16 \%)$ & $4(25 \%)$ & $1.7(0.5-6.5)$ & 0.415 \\
\hline$(-)$ & $58(83 \%)$ & $6(75 \%)$ & & & 52 (84\%) & 12 (75\%) & & \\
\hline
\end{tabular}

All results were adjusted for age and sex

Abbreviations: SS-16 the 16-item odor identification test from Sniffin Sticks 
Table 5 Analysis of potential predictors in populations with or without ApoE ع4carrier in three-years follow up

\begin{tabular}{|c|c|c|c|c|c|c|c|c|}
\hline & \multicolumn{4}{|c|}{ ApoE $\varepsilon 4$ carriers $(n=14)$} & \multicolumn{4}{|c|}{ ApoE $\varepsilon 4$ non-carriers $(n=64)$} \\
\hline & $\mathrm{MCl}-\mathrm{nc}(n=10)$ & $\mathrm{MCl}-\mathrm{nc}(n=4)$ & OR $(95 \% \mathrm{Cl})$ & $P$ & $\mathrm{MCl}-\mathrm{nc}(n=52)$ & $\mathrm{MCl}-\mathrm{nc}(n=12)$ & OR $(95 \% \mathrm{Cl})$ & $P$ \\
\hline \multicolumn{9}{|l|}{ SS-16 } \\
\hline$<8$ & 6 & 4 & / & / & 21 & 10 & $0.14(0.03-0.7)$ & 0.015 \\
\hline$\geq 8$ & 4 & 0 & & & 31 & 2 & & \\
\hline \multicolumn{9}{|c|}{$\mathrm{A} \beta_{1-42}, \mathrm{pg} / \mathrm{ml}$} \\
\hline$<14.02$ & 7 & 1 & $7(0.5-97.8)$ & 0.15 & 34 & 2 & $9.4(1.9-47.8)$ & 0.007 \\
\hline$\geq 14.02$ & 3 & 3 & & & 18 & 10 & & \\
\hline
\end{tabular}

All results were adjusted for age and sex

Abbreviations: SS-16 the 16-item odor identification test from Sniffin Sticks

it predicts the conversion of MCI to AD dementia [28]. In autopsy studies [29], the absence of odor identification was associated with plaques and tangles in the olfactory bulb, entorhinal cortex and cornu ammonis 1 regions of the hippocampus. The SS-16 test was validated as a diagnostic tool for AD dementia and MCI patients in our study. In this study, level of $A \beta_{1-42}$ in NDEs increased the risk of developing AD dementia in individuals with $\mathrm{MCI}$ in the 2 and 3-year follow-up. Compared to the 2-year follow-up, the level of $A \beta_{1-42}$ showed better predictive power for the cognitive decline in MCI individuals in a 3-year revisit. It was believed that the combination of P-tau and $A \beta_{1-42}$ in CSF had the greatest predictive accuracy for predicting the conversion from $\mathrm{MCI}$ to dementia [30,31]. A recent study also showed that plasma neuronal-derived exosomal $\mathrm{A} \beta_{1-42}, \mathrm{~T}$-tau, and P-T181-tau had the same capacity as those in CSF for the diagnosis of AD dementia and $\mathrm{MCI}$ [32].

In our study, plasma NDEs $A \beta_{1-42}$ differentiated between cognitive controls, MCI patients and AD dementia patients; and predicted the risk of MCI progressing to $\mathrm{AD}$ dementia in the longitudinal study. Neuronal exosomes containing $A \beta$-peptide products transmit $A \beta$ to adjacent cells, other brain regions and circulatory systems, indicating that neuronal exosomes extracted from

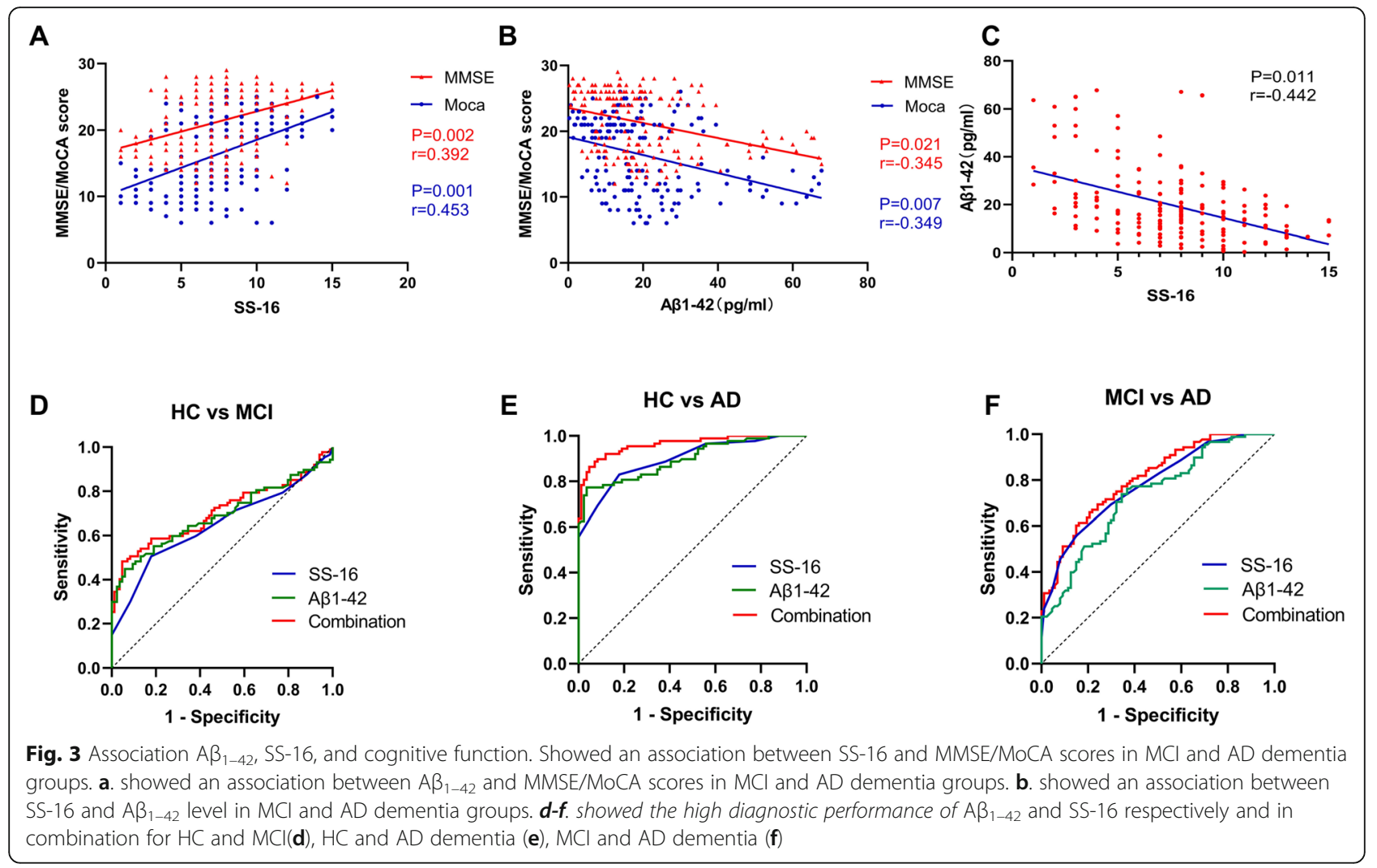


plasma or CSF can specifically evaluate the relevant neuropathological processes in the CNS [33]. Moreover, NDEs may act as vehicles for the neuron-to-neuron transfer of $A \beta$ oligomers in a prion-like manner [34]. The propagation of $A \beta$ in the brain from NDEs could also serve as a potential treatment target by inhibiting either formation, secretion, or cellular uptake of exosomes [35]. Our findings suggested that a combination of the SS-16 with that plasma NDEs $A \beta_{1-42}$ helped screen cognitively healthy individuals and MCI patients. Our findings identify that these biomarkers may be beneficial in identifying at risk individuals which may be helpful for the development of preventative medicine. The mechanisms underlying the association between exosomes and olfactory function are complex. Many studies have shown that the relationship between $A \beta$ alters the connectivity of the peripheral olfactory neural circuit even before the onset of amyloid plaques [36]. The oligomeric amyloid- $\beta$ peptide affects the responses of mitral cells (MCs) in the rat olfactory bulb [37]. Impaired bloodbrain barrier (BBB) may lead to disruptions in CSF flow through the olfactory system, resulting in the less efficient removal of $A \beta$ from the CNS [38]. What's more, exosomes played an important role in amyloid $A \beta$ clearance in CNS [39]. Hence, further research is needed to understand the relationship between exosomes and olfactory function.

Some limitations of our study should be considered when interpreting the results. First, we chose to use only one odor identification test to make the study more clinically feasible. Previous studies have indicated that patients with $\mathrm{AD}$ dementia have a higher olfactory threshold compared to healthy controls [40]. For individuals with extremely high olfactory thresholds, the odor discrimination test results may also be affected. Therefore, if conditions permit, it would be preferable to administer all three parts of the standard SS-16 to obtain the maximum amount of reliable data to fully evaluate olfactory function. Secondly, $A \beta_{1-40}$ in NDEs revealed no significant difference between the MCI and healthy controls, possibly due to the small number of cases. To further the understanding of how exosomes predict MCI transformation, future studies using larger sample sizes are needed. What's more, it is needed to verify whether the predictive power of SS-16 and NDE A $1-42$ is specific to $\mathrm{AD}$ dementia, considering that $\mathrm{MCI}$ is also associated with other kinds of dementia, such as dementia with Lewy bodies (DLB). Hence, it is necessary to include a relatively large sample size and used a longitudinal design and long time-points to verify the prediction of olfaction and A $\beta 1-42$ in NDEs with respect to conversion toward AD dementia or DLB in MCI patients.
In conclusion, our findings suggest that impaired olfaction and plasma NDE cargo proteins traffic from the CNS to blood are shown in MCI and they predict the conversion from $\mathrm{MCI}$ to $\mathrm{AD}$ dementia. Our findings highlight the clinical utility of these biomarkers to identifying at risk individuals. Further work is needed to identify whether modulating these early abnormalities may prevent the progression of $\mathrm{MCI}$ to $\mathrm{AD}$ dementia.

\section{Supplementary information}

Supplementary information accompanies this paper at https://doi.org/10. 1186/s40035-020-00210-5.

Additional file 1: Table S1. Single-question-score of SS-16 among groups in baseline. Table S2. Characteristics of ROC curves of SS-16 and A $\beta 1-42$ in NDEs among groups. Table S3. Single-question-score of SS16 between convertors and non-convertors in three-years follow up.

\section{Code availability}

Not applicable.

\section{Authors' contributions}

YD and $J$ designed the study, provided financial support and revised the manuscript. WK revised the manuscript. AZ, YL, YY, YQ, BL, WX and $Y W$ collected the data. AZ, YL and $Y Y$ carried out the follow-up visits and performed data analysis. AZ wrote the manuscript. All the co-authors contributed to revising the manuscript for intellectual content and approved the final version for publication.

\section{Funding}

This work was supported by grants from the National Natural Science Foundation of China (NSF) $(81571029,81873778,81501097)$ and the Natural Science Foundation of Shanghai (Shanghai Natural Science Foundation) (19ZR1432500). This study was also supported by grants from the National Key Research and Development Program (2016YFC1306505) and the Shanghai Municipal Education Commission-Basic Key Project (18JC1420300).

\section{Availability of data and materials}

All data generated or analysed during this study are included in this published article.

\section{Ethics approval and consent to participate}

This study was approved by the Research Ethics Committee of Ruijin Hospital.

Written informed consent was obtained from individual or guardian participants

\section{Consent for publication}

Not applicable.

\section{Competing interests}

There are no potential conflicts of interest.

\section{Author details}

${ }^{1}$ Department of Neurology and Institute of Neurology, Ruijin Hospital affiliated to Shanghai Jiao Tong University School of Medicine, Shanghai, China. ${ }^{2}$ Department of Neurology, RuiJin Hospital/LuWan Branch, School of Medicine, Shanghai Jiaotong University, Shanghai, China.

Received: 9 March 2020 Accepted: 3 July 2020

Published online: 03 August 2020

\section{References}

1. Albert MS, DeKosky ST, Dickson D, Dubois B, Feldman HH, Fox NC, et al. The diagnosis of mild cognitive impairment due to Alzheimer's disease: recommendations from the National Institute on Aging-Alzheimer's 
Association workgroups on diagnostic guidelines for Alzheimer's disease. Alzheimers Dement. 2011;7(3):270-9.

2. Petersen RC, Lopez O, Armstrong MJ, Getchius TSD, Ganguli M, Gloss D, et al. Practice guideline update summary: mild cognitive impairment: report of the guideline development, dissemination, and implementation Subcommittee of the American Academy of neurology. Neurology. 2018;90(3):126-35.

3. Hu C, Yu D, Sun X, Zhang M, Wang L, Qin $H$. The prevalence and progression of mild cognitive impairment among clinic and community populations: a systematic review and meta-analysis. Int Psychogeriatr. 2017; 29(10):1595-608.

4. Devanand DP, Lee S, Manly J, Andrews H, Schupf N, Doty RL, et al. Olfactory deficits predict cognitive decline and Alzheimer dementia in an urban community. Neurology. 2015;84(2):182-9.

5. Devanand DP, Tabert MH, Cuasay K, Manly JJ, Schupf N, Brickman AM, et al. Olfactory identification deficits and $\mathrm{MCl}$ in a multi-ethnic elderly community sample. Neurobiol Aging. 2010;31(9):1593-600

6. Devanand DP. Olfactory identification deficits, cognitive decline, and dementia in older adults. Am J Geriatr Psychiatry. 2016;24(12):1151-7.

7. Frye RE, Schwartz BS, Doty RL. Dose-related effects of cigarette smoking on olfactory function. Jama. 1990;263(9):1233-6.

8. Lee JC, Kim SJ, Hong S, Kim Y. Diagnosis of Alzheimer's disease utilizing amyloid and tau as fluid biomarkers. Exp Mol Med. 2019;51(5):53.

9. van Rossum IA, Vos S, Handels R, Visser PJ. Biomarkers as predictors for conversion from mild cognitive impairment to Alzheimer-type dementia: implications for trial design. J Alzheimers Dis. 2010;20(3):881-91.

10. Soares HD, Chen Y, Sabbagh M, Roher A, Rohrer A, Schrijvers E, et al. Identifying early markers of Alzheimer's disease using quantitative multiplex proteomic immunoassay panels. Ann N Y Acad Sci. 2009;1 180:56-67.

11. Rajendran L, Honsho M, Zahn TR, Keller P, Geiger KD, Verkade P, et al. Alzheimer's disease beta-amyloid peptides are released in association with exosomes. Proc Natl Acad Sci U S A. 2006;103(30):11172-7.

12. Fiandaca MS, Kapogiannis D, Mapstone M, Boxer A, Eitan E, Schwartz JB, et al. Identification of preclinical Alzheimer's disease by a profile of pathogenic proteins in neurally derived blood exosomes: A case-control study. Alzheimers Dement. 2015;11(6):600-7 e1.

13. McKhann GM, Knopman DS, Chertkow H, Hyman BT, Jack CR, Kawas CH, et al. The diagnosis of dementia due to Alzheimer's disease: recommendations from the National Institute on Aging-Alzheimer's Association workgroups on diagnostic guidelines for Alzheimer's disease. Alzheimers Dement. 2011:7(3):263-9.

14. Petersen RC, Smith GE, Waring SC, Ivnik RJ, Tangalos EG, Kokmen E. Mild cognitive impairment: clinical characterization and outcome. Arch Neurol. 1999;56(3):303

15. Petersen RC. Mild cognitive impairment as a diagnostic entity. J Intern Med. 2004;256(3):183-94.

16. Mungas D. In-office mental status testing: a practical guide. Geriatrics. 1991; 46(7):54.

17. Caselli RJ, Dueck AC, Locke DE, Hoffman-Snyder CR, Woodruff BK, Rapcsak SZ, et al. Longitudinal modeling of frontal cognition in APOE $\varepsilon 4$ homozygotes, heterozygotes, and noncarriers. Neurology. 2011;76(16):1383-8.

18. Ng QX, Koh SSH, Chan HW, Ho CYX. Clinical use of Curcumin in depression: a meta-analysis. J Am Med Dir Assoc. 2017;18(6):503-8.

19. Chen W, Chen S, Kang WY, Li B, Xu ZM, Xiao Q, et al. Application of odor identification test in Parkinson's disease in China: a matched case-control study. J Neurol Sci. 2012;316(1-2):47-50

20. Shi M, Liu C, Cook TJ, Bullock KM, Zhao Y, Ginghina C, et al. Plasma exosomal a-synuclein is likely CNS-derived and increased in Parkinson's disease. Acta Neuropathol. 2014;128(5):639-50.

21. Fauré J, Lachenal G, Court M, Hirrlinger J, Chatellard-Causse C, Blot B, et al. Exosomes are released by cultured cortical neurones. Mol Cell Neurosci. 2006;31(4):642-8.

22. Kapogiannis D, Boxer A, Schwartz JB, Abner EL, Biragyn A, Masharani U, et al. Dysfunctionally phosphorylated type 1 insulin receptor substrate in neural-derived blood exosomes of preclinical Alzheimer's disease. FASEB J. 2015;29(2):589-96.

23. Parker $C B$, Delong ER. ROC methodology within a monitoring framework. Stat Med. 2003;22(22):3473

24. Csukly G, Sirály E, Fodor Z, Horváth A, Salacz P, Hidasi Z, et al. The differentiation of amnestic type $\mathrm{MCl}$ from the non-amnestic types by structural MRI. Front Aging Neurosci. 2016;8:52.
25. van der Pol E, Coumans FA, Grootemaat AE, Gardiner C, Sargent IL, Harrison $P$, et al. Particle size distribution of exosomes and microvesicles determined by transmission electron microscopy, flow cytometry, nanoparticle tracking analysis, and resistive pulse sensing. J Thromb Haemost. 2014;12(7):1182-92.

26. Li W, Howard JD, Gottfried JA. Disruption of odour quality coding in piriform cortex mediates olfactory deficits in Alzheimer's disease. Brain. 2010;133(9):2714-26.

27. Petersen RC, Doody R, Kurz A, Mohs RC, Morris JC, Rabins PV, et al. Current concepts in mild cognitive impairment. Arch Neurol. 2001;58(12):1985-92.

28. Roberts RO, Christianson TJ, Kremers WK, Mielke MM, Machulda MM, Vassilaki M, et al. Association between olfactory dysfunction and amnestic mild cognitive impairment and Alzheimer disease dementia. JAMA Neurol. 2016;73(1):93-101.

29. Wilson RS, Arnold SE, Schneider JA, Tang Y, Bennett DA. The relationship between cerebral Alzheimer's disease pathology and odour identification in old age. J Neurol Neurosurg Psychiatry. 2007;78(1):30-5.

30. Blennow K, Hampel H. CSF markers for incipient Alzheimer's disease. Lancet Neurol. 2003;2(10):605-13.

31. Lanari A, Parnetti L. Cerebrospinal fluid biomarkers and prediction of conversion in patients with mild cognitive impairment: 4-year follow-up in a routine clinical setting. ScientificWorldJournal. 2009;9:961-6.

32. Jia L, Qiu Q, Zhang H, Chu L, Du Y, Zhang J, et al. Concordance between the assessment of Aß42, T-tau, and P-T181-tau in peripheral blood neuronal-derived exosomes and cerebrospinal fluid. Alzheimers Dement. 2019;15(8):1071-80.

33. Jaunmuktane Z, Mead S, Ellis M, Wadsworth JD, Nicoll AJ, Kenny J, et al. Evidence for human transmission of amyloid- $\beta$ pathology and cerebral amyloid angiopathy. Nature. 2015;525(7568):247-50.

34. Sardar Sinha M, Ansell-Schultz A, Civitelli L, Hildesjö C, Larsson M, Lannfelt L, et al. Alzheimer's disease pathology propagation by exosomes containing toxic amyloid-beta oligomers. Acta Neuropathol. 2018;136(1):41-56.

35. Dinkins MB, Dasgupta S, Wang G, Zhu G, Bieberich E. Exosome reduction in vivo is associated with lower amyloid plaque load in the 5XFAD mouse model of Alzheimer's disease. Neurobiol Aging. 2014;35(8):1792-800.

36. Cao L, Schrank BR, Rodriguez S, Benz EG, Moulia TW, Rickenbacher GT, et al. $A \beta$ alters the connectivity of olfactory neurons in the absence of amyloid plaques in vivo. Nat Commun. 2012;3:1009.

37. Hu B, Geng C, Hou XY. Oligomeric amyloid- $\beta$ peptide disrupts olfactory information output by impairment of local inhibitory circuits in rat olfactory bulb. Neurobiol Aging. 2017;51:113-21.

38. Ethell DW. Disruption of cerebrospinal fluid flow through the olfactory system may contribute to Alzheimer's disease pathogenesis. J Alzheimers Dis. 2014;41(4):1021-30.

39. Lim CZJ, Zhang Y, Chen Y, Zhao H, Stephenson MC, Ho NRY, et al. Subtyping of circulating exosome-bound amyloid beta reflects brain plaque deposition. Nat Commun. 2019;10(1):1144

40. Murphy C, Gilmore MM, Seery CS, Salmon DP, Lasker BR. Olfactory thresholds are associated with degree of dementia in Alzheimer's disease. Neurobiol Aging. 1990;11(4):465-9.

\section{Ready to submit your research? Choose BMC and benefit from:}

- fast, convenient online submission

- thorough peer review by experienced researchers in your field

- rapid publication on acceptance

- support for research data, including large and complex data types

- gold Open Access which fosters wider collaboration and increased citations

- maximum visibility for your research: over $100 \mathrm{M}$ website views per year

At $\mathrm{BMC}$, research is always in progress.

Learn more biomedcentral.com/submission 